\section{GENOMICS}

\section{Spoilt for choice}

Rhodopseudomonas palustris is a purple photosynthetic bacterium that can be isolated from many diverse environmental locations. The key to success for this microorganism is its extraordinary metabolic versatility $R$. palustris can exploit light, inorganic compounds and organic compounds for energy. It can also acquire carbon from organic componds and by $\mathrm{CO}_{2}$ fixation, grow with or without oxygen, and can fix nitrogen. Now, with the publication of the R. palustris genome in Nature Biotechnology, an important step has been taken towards understanding how this bacterium coordinates its many metabolic capabilities in response to changing environmental conditions.

The genome of R. palustris comprises a chromosome 5.46 Mb in size and a 8.4-kb circular plasmid. The sequence reveals the presence of a large number of genes that allow the microorganism to benefit from considerable flexibility within a given type of metabolism and to take advantage of changes in available carbon, nitrogen, light and oxygen. Genes for three different nitrogenases, multiple aromatic degradation pathways, four light harvesting (LH)-2 complexes and multiple oxidoreductases were discovered. $R$. palustris also encodes about 325 transport systems, devoting $15 \%$ of the genome to this activity. In other bacterial genomes, typically 5-6\% of a genome consists of transport genes, indicating that $R$. palustris is richly equipped to sense and acquire diverse compounds from its environment. Of course, with so many metabolic options, it is crucial that the bacterium can sense changing environmental conditions rapidly and regulate gene expression appropriately for survival and growth. To this end, R. palustris dedicates $9.3 \%$ of its genome to regulation (versus 5-6\% for most free-living bacteria), including 63 signal transduction kinases and 79 response regulator receiver domains.

The availability of the R. palustris genome provides the framework that will allow researchers to explore the regulatory strategies employed by the microorganism to select and integrate its many metabolic capabilities. By understanding, and ultimately manipulating these processes, the full biotechnological potential of $R$. palustris can be realized, including the development of the bacterium as a biocatalyst for hydrogen production, and

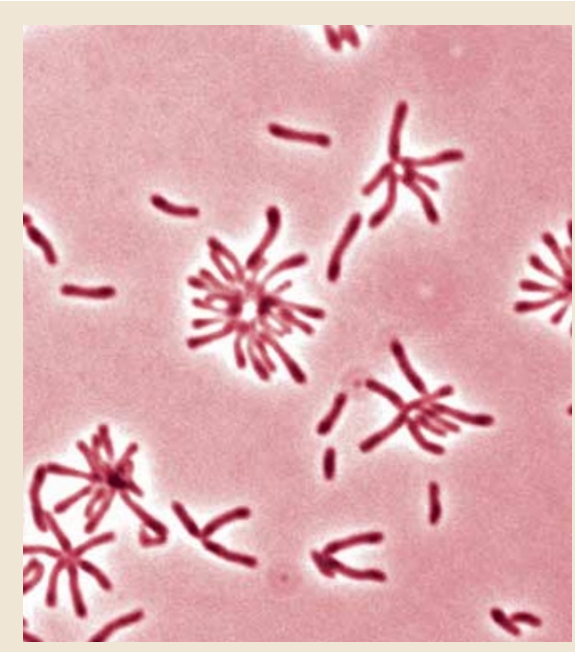

Photomicrograph, kindly provided by Caroline Harwood (University of lowa, USA), shows Rhodopseudomonas palustris cells.

devising strategies to maximize its promise as an effective aid to environmental restoration through microbially mediated bioremediation.

David O'Connell

\section{(2) References and links}

ORIGINAL RESEARCH PAPER Larimer, F. W. et al. The genome sequence of the metabolically versatile photosynthetic bacterium Rhodopseudomonas palustris. Nature Biotechnol. (2003) doi:10.1038/nbt923

\title{
Cattle vaccine for deadly E. coli strain
}

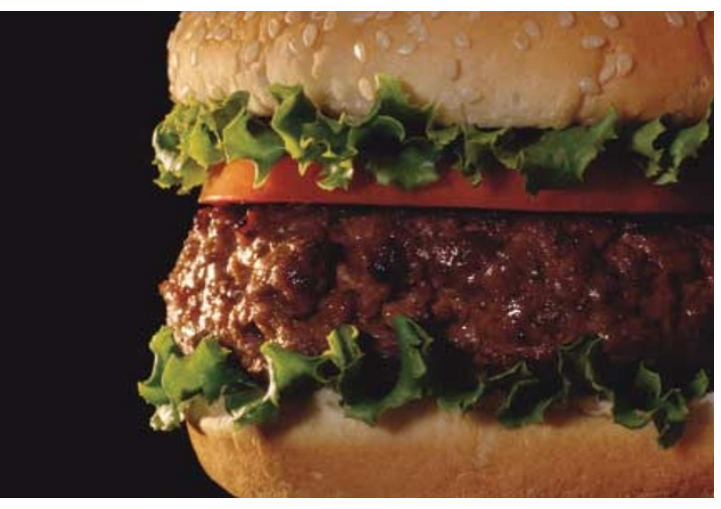

Escherichia coli serotype O157:H7 causes illness in thousands of humans every year, and can be fatal. Results of a recent study published in the journal Vaccine now indicate that immunizing livestock against this bacterium could provide an effective method of minimizing its spread.

Although most people affected by E. coli O157:H7 experience only 'food poisoning' symptoms, some cases result in potentially lethal kidney failure. Most cases of human infection can be traced back to cattle through consumption of beef products or contaminated water, suggesting that vaccination of animals against the bacterium might decrease the number of people infected.

Brett Finlay and colleagues immunized eight calves with a vaccine containing the proteins EspA and Tir - part of a type III secretion system that is required for E. coli O157:H7 to colonize the host gut. Vaccinated animals showed a 45 -fold increase in the production of specific antibodies against these proteins when an initial immunization was followed by a booster shot at 21 days. In addition, after experimental infection with the pathogen, the number of bacteria shed in faeces - a major route for the spread of infection between animals - was significantly lower in vaccinated calves than in animals that were given a placebo vaccine.

On the basis of these promising results, a second trial was carried out on yearling cattle. Finlay and co-workers found that the numbers of vaccinated cattle shedding O157:H7 fell to zero 6 days after infection, whereas $\sim 70 \%$ of animals that were administered a placebo were still shedding the bacterium at the same time-point. In addition, the number of bacteria present in faeces was markedly lower in the vaccinated animals.

Finally, the authors tested the effects of vaccination of a cattle population that were exposed to E. coli $\mathrm{O} 157: \mathrm{H} 7$ infection by natural means under typical agricultural conditions. A significant reduction in the shedding of the pathogen was seen among vaccinated cattle, with $8.8 \%$ of these animals releasing the bacterium in their faeces as compared with $21.3 \%$ of unvaccinated animals.

The study also suggested that just two rounds of immunization are likely to provide a sufficient level of protection to significantly reduce the spread of the bacterium, although further tests are required to confirm this. Together with the fact that the vaccine used is simple and cheap to produce, this highlights the immunization of cattle as a promising, economically viable method for curbing the number of animals infected with $E$. coli O157:H7 and lowering the risk of spread to the human population.

Louisa Flintoft

\section{(D) References and links}

ORIGINAL RESEARCH PAPER Decreased shedding of

Escherichia coli $\mathrm{O} 157: \mathrm{H} 7$ by cattle following vaccination with type III secreted proteins. Potter, A. A. et al. Vaccine (2003).

doi: 10.1016/j.vaccine.2003.08.007 